\title{
A Space Weather mission concept: Observatories of the Solar Corona and Active Regions (OSCAR)
}

Antoine Strugarek $^{1,2, *}$, Nils Janitzek ${ }^{3}$, Arrow Lee ${ }^{4}$, Philipp Löschl ${ }^{5}$, Bernhard Seifert ${ }^{6}$, Sanni Hoilijoki ${ }^{7,8}$, Emil Kraaikamp ${ }^{9}$, Alankrita Isha Mrigakshi ${ }^{3,10}$, Thomas Philippe ${ }^{11}$, Sheila Spina ${ }^{12}$, Malte Bröse ${ }^{13}$, Sonny Massahi ${ }^{14}$, Liam O’Halloran ${ }^{15}$, Victor Pereira Blanco ${ }^{16}$, Christoffer Stausland ${ }^{17}$, Philippe Escoubet $^{18}$, and Günter Kargl ${ }^{19}$

1 Département de Physique, Université de Montréal, C.P. 6128 Succ. Centre-Ville, Montréal, QC, H3C-3J7, Canada

*Corresponding author: strugarek@astro.umontreal.ca

2 Laboratoire AIM Paris-Saclay, CEA/Irfu Université Paris-Diderot CNRS/INSU, 91191 Gif-sur-Yvette, France

3 Institute of Experimental and Applied Physics, University of Kiel, Kiel, Germany

4 Mullard Space Science Laboratory, University College London, Dorking, United Kingdom

5 Institut für Astrophysik, University of Vienna, Vienna, Austria

6 FOTEC - Department of Aerospace Engineering, Wiener Neustadt, Austria

Finnish Meteorological Institute, Helsinki, Finland

Department of Physics, University of Helsinki, Helsinki, Finland

Royal Observatory of Belgium, Brussels, Belgium

German Aerospace Centre (DLR), Institute of Aerospace Medicine, Cologne, Germany

11 Institut Supérieur de l'Aéronautique et de l'Espace, Toulouse, France

12 La Sapienza - University of Rome, Italy

13 Department of Physics, Free University of Berlin, Berlin, Germany

14 Danish Space Research Institute, Copenhagen, Denmark

15 School of Medicine and Medical Sciences, University College Dublin, Dublin, Ireland

16 Dpto. de Astrofísica y CC. de la Atmósfera, Universidad Complutense de Madrid, 28040 Madrid, Spain

17 Department of Physics, University of Oslo, Norway

18 ESA/ESTEC, Noordwijk, The Netherlands

19 Space Research Institute, Austrian Academy of Sciences, Austria

Received 28 February 2014 / Accepted 1 September 2014

\begin{abstract}
Coronal Mass Ejections (CMEs) and Corotating Interaction Regions (CIRs) are major sources of magnetic storms on Earth and are therefore considered to be the most dangerous space weather events. The Observatories of Solar Corona and Active Regions (OSCAR) mission is designed to identify the 3D structure of coronal loops and to study the trigger mechanisms of CMEs in solar Active Regions (ARs) as well as their evolution and propagation processes in the inner heliosphere. It also aims to provide monitoring and forecasting of geo-effective CMEs and CIRs. OSCAR would contribute to significant advancements in the field of solar physics, improvements of the current CME prediction models, and provide data for reliable space weather forecasting. These objectives are achieved by utilising two spacecraft with identical instrumentation, located at a heliocentric orbital distance of $1 \mathrm{AU}$ from the Sun. The spacecraft will be separated by an angle of $68^{\circ}$ to provide optimum stereoscopic view of the solar corona. We study the feasibility of such a mission and propose a preliminary design for OSCAR.
\end{abstract}

Key words. Missions - Corona - Active region - Interplanetary Coronal Mass Ejection (CME) - Corotating Interaction Regions (CIR)

\section{Introduction}

The OSCAR mission concept was conceived during the Alpbach Summer School ${ }^{1} 2013$ on space weather over a period of 2 weeks. We report here the resulting concept that, we believe, is of significant interest for the design of a future space weather oriented space mission. Our mission consists of twin satellites orbiting the Sun at $1 \mathrm{AU}$, with one leading the Earth and the other trailing behind it. They are designed to improve significantly our knowledge of space weather phenomena as well as to develop a space-based space weather forecasting system.

\footnotetext{
${ }^{1} \mathrm{http}: / /$ www.summerschoolalpbach.at/
}

This paper is organised as follows. We first give a quick introduction on the background and motivations that lead to this mission concept (Sect. 2). We then develop the mission objectives and the associated key scientific requirements of OSCAR (Sect. 3). In Section 4, we provide details of the instrumentation needed onboard OSCAR to satisfy those requirements. A preliminary design of the spacecraft for such a payload is given in Section 5. The mission design, from the orbit selection to the operational modes and the ground segment, is then described in Section 6. In the following Section 7, an estimate of the mission cost and subsequent descoping options is discussed. We finally conclude our study in Section 8 . 


\section{Background and motivation}

Space weather describes the changes in the near-Earth ambient plasma that result from solar and cosmic activity. It is a field of major importance in society today as the environmental conditions in the vicinity of Earth severely affect space- and groundbased systems (see, e.g., Schwenn 2006; Pulkkinen 2007). Most frequent space weather events originate from the Sun. In order to improve our knowledge and anticipation of space weather phenomena one needs to (i) study the origin of those events inside and at the surface of the Sun, (ii) accurately describe their propagation in the interplanetary medium from Sun to Earth and (iii) improve our understanding of how they impact Earth's magnetosphere and atmosphere.

Solar dynamics lead to the triggering of CMEs and indirectly to the development of CIRs that propagate in the solar wind. When they encounter the Earth's magnetosphere, they trigger strong magnetic reconfigurations known as geomagnetic storms. CMEs alone cause more than $80 \%$ of geomagnetic storms and thus represent a severe threat for modern technology (Zhang et al. 2007; Liu et al. 2014). CMEs originate from the complex magnetic structures within the solar ARs. These regions are created by magnetic flux tubes arising from beneath the photosphere, ultimately forming loops in the corona that are anchored to the solar photosphere (for a review, see Fan 2009). Magnetic shears and stresses within the photosphere lead to an increase of the energy stored in the coronal loops, often triggering magnetic reconnection inside them and leading to explosive events called flares which emit energetic particles and radiate in the X-ray and extreme ultra-violet (EUV) bands. In some particular configurations, CMEs are triggered and erupt from the AR (see, e.g., Nitta et al. 2013). Note that CMEs are not systematically correlated with flares (Webb \& Howard 2012), but the majority of large flares are observed to be followed by CMEs (Yashiro et al. 2005). Multiple models have been developed to pinpoint the exact triggering mechanism(s) of CMEs, but their unification is still a major challenge in solar astrophysics (see Zuccarello et al. 2013, and references therein). Such unification would greatly help space weather forecasting programmes to anticipate CME launches from the solar surface, and discriminate the potential threats further in advance. Hence, the understanding of the trigger of CMEs is a major goal for the progress of solar physics as well as the development of space weather forecasting systems.

Once triggered, the CMEs propagate outwards from the Sun in the corona and can reach a distance of $1 \mathrm{AU}$ in a time ranging from $14 \mathrm{~h}$ up to 5 days (Chen 2011). The fast-moving CMEs, when geo-directed, are generally considered to be the most dangerous space weather events and are also the most difficult to anticipate due to their high propagation velocity. In addition, CMEs can either accelerate or decelerate during their propagation from Sun to Earth (Gopalswamy et al. 2000) depending on the ambient solar wind in which they evolve and on their initial energy (Manoharan 2006) it. Hence, forecasts have to use either well-cadenced observations of the heliosphere or solar wind models that accurately take the acceleration/deceleration processes into account. The best well-cadenced heliospheric data today comes from the Solar Terrestrial Relations Observatory (STEREO) mission which proved to be very useful for the prediction of CME arrival time at Earth. The ability to combine direct images of the heliosphere from the Sun to $1 \mathrm{AU}$ from different points of view with the STEREO spacecraft led to significant novel capabilities in the context of space weather forecasting. To cite a few,
Möstl et al. (2014) showed that the use of heliospheric images was extremely effective in reconstructing and forecasting highspeed solar wind streams. Using the stereoscopic capabilities, Davies et al. (2013) recently developed a robust technique to determine time profiles and propagation direction of transients in the solar wind. These observations were even shown to provide the capability to determine the excitation site of solar energetic particles observed at $1 \mathrm{AU}$ and to relate this site to a particular CME event (see, e.g., Rouillard et al. 2012). However, because of the choice of orbits for the two STEREO satellites, the data best suited for stereoscopic observations is available for a very limited amount of time. Hence, no reliable long-term forecasting system of CMEs can be set up from those observations.

CIRs are produced when the fast solar wind catches up with the slow wind. They consist of high pressure regions that co-rotate with the Sun in the solar wind. They eventually lead to the formation of co-rotating shocks in the supersonic wind, generally at distances larger than 2 AU (Richardson 2004). Depending on their magnetic field orientation with respect to the Earth's magnetic field, CIRs can transfer part of their energy to the magnetosphere which, in turn, causes weak to moderate magnetic storms. Some of these storms can cause significant damage, not only to space technology but also to communication, transportation and electrical power systems.

There are currently missions like STEREO and the Solar Dynamics Observatory (SDO) which aim to study - at least some aspects of - CMEs and CIRs. While these missions are still providing important new insights for our understanding of the solar corona, none of them truly tackle the hard task of providing a space-based, reliable and long-term space weather forecasting system. In addition, STEREO's stereographic images demonstrated the large gain we obtain from observing the Sun with simultaneous different points of view (for recent reviews, see Bemporad 2009; Zuccarello et al. 2013, and references therein). Hereby we propose a new mission concept, OSCAR, which aims to provide new and decisive stereoscopic data that will allow us to finally identify how CMEs are triggered and how to forecast them. With our mission design, for the first time and during its whole lifetime, an efficient forecast of geo-effective CMEs and CIRs will be operating in nearreal time. Taking advantage of the position of the spacecraft, CMEs will be monitored and forecasted with remote-sensing instruments and CIRs will be forecasted thanks to in-situ measurements before reaching the Earth.

\section{Combining science objectives with near-real time forecasts}

The OSCAR mission addresses the difficult challenge of space weather forecasting, from the initiation of interplanetary CMEs (hereafter generically referred to as CMEs) to their coupling with Earth's magnetosphere. In this context, we are interested in the most energetic CMEs and CIRs that can affect the terrestrial environment and human life. In this section we define the mission objectives (Sect. 3.1) as well as the associated scientific key requirements (Sect. 3.2).

\subsection{Mission objectives}

We break down the mission objectives into one primary objective and two secondary objectives. 
1. Unveil the trigger mechanism(s) of CMEs in active regions and their robust forecasting indices

The primary objective is to provide data to efficiently forecast the onset of CMEs in active regions on the solar surface. This has to be achieved through the study of the 3D structure of coronal loops to unveil the physical trigger mechanism(s) of CMEs. The highly energetic CMEs that we are interested in are strongly associated with M- and X-class flares (only $10 \%$ of the X-class flares are not associated with CMEs, see Yashiro et al. 2005). We will observe the magnetic field of sunspots, the 3D structure of coronal loops and the flaring process in ARs at the onset of CMEs. We know that flares and their associated CMEs (when these are present) occur on a time scale from minutes to hours (Shibata \& Magara 2011). High-cadenced observations - one of the major challenges for this mission - of these three quantities will allow us to sample the whole eruption process with unprecedented details. This will in turn provide strong constraints on the various physical models of solar eruptions (Zuccarello et al. 2013) and allow the identification of key plasma quantities as robust trackers of the trigger of CMEs. In addition, we will observe hundreds of ARs during the lifetime of OSCAR and be able to create a catalogue of AR topologies, associated with their ability to trigger CMEs. This catalogue will be used to validate theoretical models and numerical simulations in terms of necessary conditions - e.g., in terms of magnetic helicity in the magnetic structure of the AR or temperature profiles across the flux tubes - for the trigger of a CME. Hence, it will be highly valuable to further guide the future forecasting of CME triggers on the Sun.

2.a Provide the necessary data for a near-real time forecasting geo-affecting/effective CMEs and CIRs

Real-time estimates of arrival time for geo-effective CMEs remain rather inaccurate today (Davis et al. 2011) and strongly depend on solar wind models. The aim of OSCAR is to provide data for accurate prediction of arrival time of those CMEs (either with a data-driven or a purely empirical model, e.g. Howard \& Tappin 2008).

CIRs produce $13 \%$ of geomagnetic storms (Zhang et al. 2007). Because they can last a long time and even repeat themselves after a 27-day solar rotation, they represent a threat for space-based infrastructure (Borovsky \& Denton 2006). In-situ measurements are consequently required to observe them. Since they co-rotate with the Sun, their propagation speed requires distant in-situ observation to be able to forecast them. With OSCAR, we intend to provide data for reliable forecasts of CIRs.

Altogether the OSCAR mission will provide 6-h updated forecasting data of ocurring CMEs based on high-cadenced remote-sensing measurements and of CIRs based on well-located in-situ measurements. This will ensure a forecasting window of about $8 \mathrm{~h}$ for the fastest CMEs and of 2 full days for CIRs. Thus, OSCAR will sustain for all its lifetime a reliable forecasting for CMEs and CIRs. 2.b Enhance our understanding of spatial structures of CMEs and CIRs at $1 \mathrm{AU}$

Our knowledge of the composition, geometry and magnetic field of geo-effective CIRs and CMEs is mainly based on local measurements in the vicinity of Earth (except for the STEREO measurements). With the OSCAR mission we will be able to cover an angle of $68^{\circ}$ for CME in-situ measurements at 1 AU. In addition, OSCAR will be able to trace the evolution of CIRs on a short time scale of 5.5 days, which was shown by STEREO to be the shortest typical time scale for major changes in the solar wind structure (Gómez-Herrero et al. 2011).

Combining this large set of in-situ measurements with the remote-sensing techniques described above, OSCAR shall be able to enhance our basic understanding of space weather relevant CME/CIR aspects such as (i) CME propagation out to $1 \mathrm{AU}$ (Dal Lago et al. 2013), (ii) CME shock acceleration of energetic electrons (Simnett et al. 2002), (iii) interaction of CMEs and CIRs at 1 AU (Gómez-Herrero et al. 2011) and (iv) CIR shock acceleration of low energetic ions, which were found to be an additional precursor for geomagnetic storms (although in general the shock structure of CIRs fully develops much beyond 1 AU, it was shown by Smith et al. 2004. that some inner CIRs can be fully developed and are at the origin of some large geomagnetic storms).

Finally, OSCAR also provides numerous targets of opportunity for studying space weather events from the lower corona to $1 \mathrm{AU}$ with multiple line of sight observations and multiple in-situ measurements. We choose to focus here on the main objectives of OSCAR, which are directly related to the forecast of space weather events (see list above) to clearly outline the feasibility of this mission concept. We leave a more exhaustive objectives list - with more science-oriented aspects - for further studies.

\subsection{Scientific requirements}

In order to fulfil our objectives, we detail the scientific requirements for each of them in Table 1. We separate (top level) global requirements from (second level) quantitative requirements. We give hereafter short justifications for some key requirements, which will be used to identify the instruments required for OSCAR (Sect. 4).

The stereographic observations (required for any $3 \mathrm{D}$ reconstruction of coronal loop structures) are one of the cornerstones of our mission. Thanks to the experience gained from STEREO, we can precisely define an optimal separation angle between the two lines of view for the three-dimensional reconstruction of coronal loops in an AR. Aschwanden et al. (2012) demonstrated that the quality of a stereoscopic reconstruction depends on both (i) the quality of correspondance between the two images and (ii) the accuracy of the triangulation process. The former decreases as the separation angle decreases while the latter is maximised for an angle of $90^{\circ}$. The product of the two factors gives the overall quality factor of the reconstruction that is given in Figure 1. An optimal angle is found around $68^{\circ}$ and an acceptable stereoscopic reconstruction is obtained at a separation angle between $22^{\circ}$ and $125^{\circ}$. The onset 
Table 1. Scientific requirements for the OSCAR mission.

\begin{tabular}{ll}
\hline \hline Objective & \multicolumn{1}{c}{ Top level requirements } \\
\hline $\begin{array}{l}\text { 1 - Trigger mechanism(s) of } \\
\text { forecasting indices }\end{array}$ & $\begin{array}{l}\text { Stereographic view of coronal loops at } \\
\text { different heights in the lower corona }\end{array}$ \\
& $\begin{array}{l}\text { Capture the time scale of flares and of the } \\
\text { triggering sequence of strong CMEs } \\
\text { Resolve distinct coronal loops in ARs }\end{array}$ \\
& $\begin{array}{l}\text { Synchronised stereographic images to ensure } \\
\text { a proper 3D reconstruction during the } \\
\text { eruption process }\end{array}$ \\
& $\begin{array}{l}\text { Observe the photospheric vector magnetic } \\
\text { field in ARs }\end{array}$ \\
& The duration of OSCAR shall ensure high \\
& statistics for the CME triggering \\
Track geo-directed CMEs over the whole Sun- & Earth distance
\end{tabular}

CMEs

2.a - Provide data for the forecast of geo-effective CIRs

$2 . b$ - Measure the propagation and spatial expansion of CMEs out to $1 \mathrm{AU}$

2.b - Measure the evolution of CIR spatial structures at $1 \mathrm{AU}$
Determine the shape, direction and velocity of the leading edge of the CME (common with 2.b)

Sufficient data to forecast the arrival time of all geo-directed CMEs

In-situ measurements of geo-affecting CIRs

before they reach Earth

Guarantee sufficient warning time

In-situ measurements of the magnetic field (common with 2.b)

Measurement of the in-situ solar wind proton plasma parameters (speed, temperature, density; common with 2.b)

Measure continuously the plasma parameters and the magnetic field at $1 \mathrm{AU}$ (in CMEs and CIRs)

Measure high energetic electrons accelerated at CME shock fronts close to the Sun

Remote-sensing and tracking of CIRs within one Carrington rotation

Multi-point observations at $1 \mathrm{AU}$ to detect changes in the structure of CIRs within one Carrington rotation

Measure low energetic ion events accelerated at CIR shock fronts at or beyond $1 \mathrm{AU}$

Second level requirements
The separation angle shall be between 22 and $125^{\circ}$, as
close to 68 degrees as possible (Fig. 1)

Time resolution of $5 \mathrm{~s}$ for coronal loop images

Spatial resolution better than $500 \mathrm{~km}$ in the solar upper transition region

The two spacecraft shall be synchronised with a precision of $0.1 \mathrm{~s}$

Spatial resolution better than $750 \mathrm{~km}$, with a precision of $0.1 \mathrm{G}$ for the longitudinal field and $20 \mathrm{G}$ for the transverse field

The duration of the mission shall be no less than 5 years

Observations from the lower corona to $1 \mathrm{AU}$ shall be possible

The cadence shall be of $2 \mathrm{~h}$ and stereoscopic view shall be available ${ }^{\mathrm{a}}$

The data shall enable a 2-day forecast updated every $6 \mathrm{~h}$

One spacecraft shall be positioned on a keplerian orbit close to 1 AU following Earth

Minimal separation between the Earth-following spacecraft and Earth of $29.7^{\circ}$ (warning time of 2.25 days)

The vector magnetic field shall be measured within the range $\pm 200 \mathrm{nT}$ with an accuracy of $0.1 \mathrm{nT}$ and an operational time resolution of $1 \mathrm{~min}^{\mathrm{b}}$

The solar wind protons shall be measured up to a speed of $1000 \mathrm{~km} / \mathrm{s}$ (with a $5 \%$ accuracy) with an operational time resolution of $1 \mathrm{~min}^{\mathrm{b}}$

The solar wind proton speed, temperature and density, the electron $3 \mathrm{D}$ velocity distribution, prominent ion charge states of $\mathrm{C}, \mathrm{O}, \mathrm{Si}, \mathrm{Fe}$ and magnetic field shall be measured every $15 \mathrm{~min}^{\mathrm{c}}$

The energetic electrons passing the spacecraft at $1 \mathrm{AU}$ in the range $40 \mathrm{keV}-300 \mathrm{keV}$ shall be measured every minute $^{\mathrm{d}, \mathrm{e}}$

CIRs shall be monitored in the heliosphere over an elongation angle of $150^{\circ}$ over 20 days (brightness sensitivity $<3 \times 10^{-16}$ the solar brightness ${ }^{\mathrm{f}}$

Minimum seperation angle of $66^{\circ}$ in longitude to catch major longitudinal changes, latitudinal separation $<5^{\circ \mathrm{g}}$

The protons and alpha particles in the energy range $50 \mathrm{keV}-4 \mathrm{MeV}$ shall be measured every minute ${ }^{\mathrm{d}, \mathrm{g}}$

${ }^{a}$ Howard \& Tappin (2008); ' Zwickl et al. (1998); ${ }^{\mathrm{c}}$ Gosling (1992), Gloeckler et al. (1998), McComas et al. (1998), Webb \& Howard (2012), Dal Lago et al. (2013); ${ }^{\mathrm{d}}$ Gold et al. (1998); ${ }^{\mathrm{e}}$ Simnett et al. (2002), Kahler et al. (2005); ${ }^{\mathrm{f}}$ Howard et al. (2008), Tappin \& Howard (2009); ${ }^{\mathrm{g}} \mathrm{Mason}$ et al. (2009), Gómez-Herrero et al. (2011).

of a CME occurs on a time scale of tens of seconds in a coronal structure. Hence, our ambitious primary objective requires high cadenced - high resolution (see Table 1) observations of coronal loops (and associated photospheric magnetic fields) in ARs.

The major requirements for our secondary objectives are (i) the ability to provide sufficient regular data for an efficient forecast and (ii) to accurately measure the propagation and expansion of CMEs (from the lower corona to $1 \mathrm{AU}$ ) and the evolution of the spatial structure of CIRs (at $1 \mathrm{AU})$ with a combination of remote-sensing and in-situ observations. The former requires relatively high-cadenced data (images of the Sun-Earth heliosphere every $2 \mathrm{~h}$ ) to ensure that the fastest CMEs that reach Earth within approximately $15 \mathrm{~h}$ can be accurately forecasted. We refer the reader to Table 1 (and references therein) for more details regarding the other specific requirements. Some requirements apply to several objectives, in which case they have not been repeated for sake of simplicity.

Anticipating the detailed design we give in Section 6, the combination of second level requirements for objectives (1) 


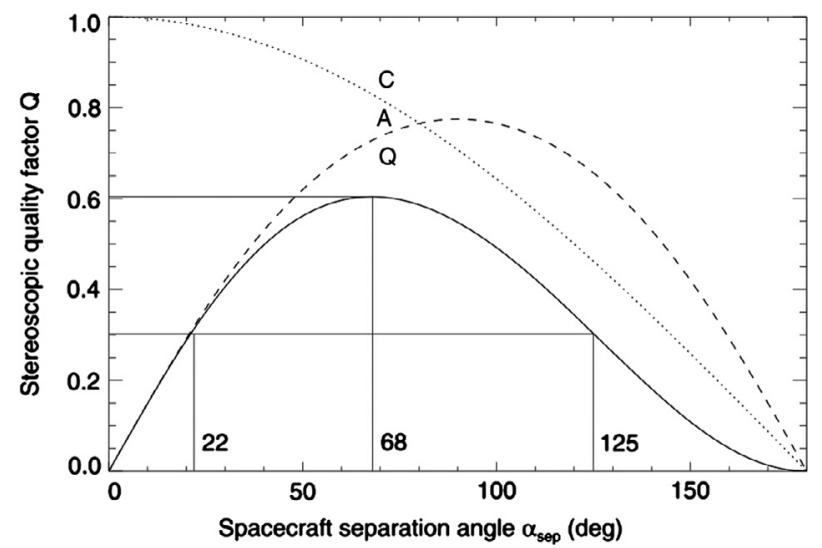

Fig. 1. Quality factor $Q$ of stereoscopic triangulation as a function of the spacecraft separation angle $\alpha_{\text {sep }}$, which is a function of the accuracy A of triangulated stereoscopic positions and the stereoscopic correspondence quality factor $\mathrm{C}$. The best quality (within a factor of 2) occurs in the range of $\alpha_{\text {sep }}=22^{\circ}-125^{\circ}$ with an optimal separation angle of $68^{\circ}$. Figure reused from Aschwanden et al. (2012) with the authorisation from Springer.

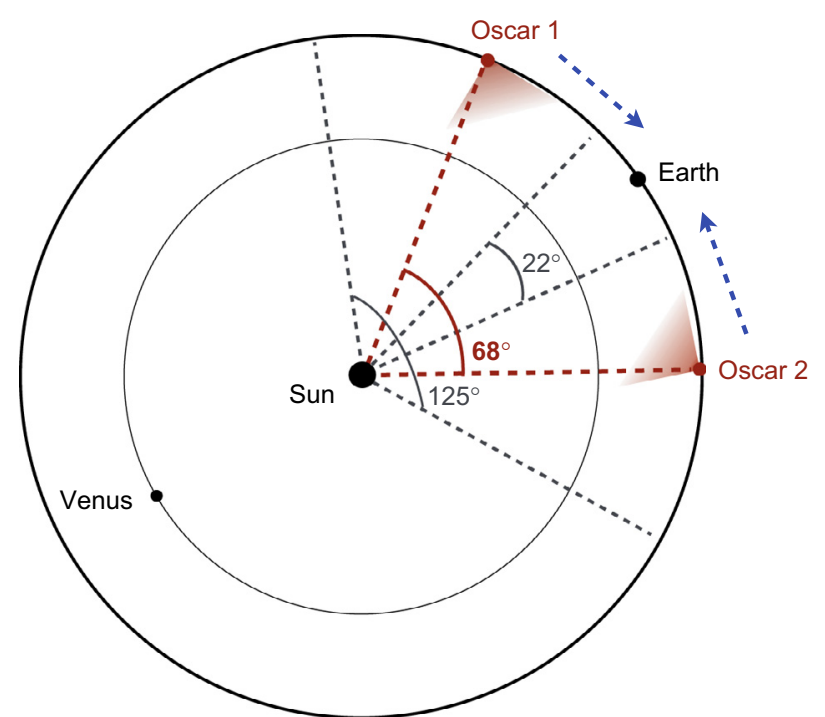

Fig. 2. Schematic of the OSCAR mission orbital configuration as viewed from the top of the ecliptic plane. The twin spacecraft orbit the Sun at $1 \mathrm{AU}$ with Oscar 1 leading the Earth and Oscar 2 trailing behind it. They are separated by an angle of $68^{\circ}$ to cover stereoscopically the photosphere, the upper transition region, the corona and the heliosphere to $1 \mathrm{AU}$ (see Sect. 6 for more details).

and (2a) suggests the use of two identical spacecraft separated by an angle of $68^{\circ}$ orbiting the Sun in the Earth orbit. Hence, one can easily imagine one spacecraft leading the Earth and the other trailing behind it with a separation angle of $34^{\circ}$. A schematic of this design is given in Figure 2. This design also fulfils all the other scientific requirements listed in Table 1. As an example, the satellite orbiting behind Earth will measure CIRs 2.25 days before they hit Earth. This design also happens to be achievable with moderate technological developments, as will be made clear in Sections 5 and 6 .

\section{Instrumentation}

Each spacecraft will carry an identical set of instruments for the purpose of investigating the Sun and the heliosphere.
A suite of telescopes will image the space between the solar surface and $1 \mathrm{AU}$ on the Sun-Earth line almost continuously. Another suite of sensors will measure the in-situ particle and magnetic field environment at 1 AU. An overview of the planned instruments, with estimates of their mass and power consumption, is given in Table 2. In order to demonstrate the feasibility of OSCAR, we particulary outline the heritage of our planned instruments. Should this mission concept be further explored, we give paths of improvements for the critical instruments of OSCAR.

\subsection{Remote-sensing instrumentation}

The package of remote-sensing instruments will consist of four instruments carrying in total six telescopes. Together they will be able to cover the photosphere, the upper transition region, the corona and the heliosphere to beyond $1 \mathrm{AU}$.

Photospheric Imager (PIM) - The Photospheric Imager will provide 2D-maps of the magnetic field vector in the photosphere by measurements of the Zeeman effect. Similar instruments like SDO's HMI have been built in the past, whereas the upcoming Solar Orbiter's Polarimetric and Heliospheric Imager (PHI) will serve as heritage (Gandorfer et al. 2011) in this case. Unlike PHI, only one telescope is required for PIM since the PHI's High-Resolution Telescope's (HRT) of $200 \mathrm{~km}$ at $0.28 \mathrm{AU}$ already fulfils the spatial resolution as well as the cadence requirements when constantly positioned at a $1 \mathrm{AU}$ orbit. PHI's restricted field of view of $16.8^{\prime}$ has to be doubled to observe the full solar disk at $1 \mathrm{AU}$, which has to be resolved in further development. With this setup PIM will be able to observe the spectral line of neutral iron at $617.3 \mathrm{~nm}$ with a spatial resolution of about $720 \mathrm{~km}$ in the photosphere and a cadence of 45-60 s, which are both within the requirements presented earlier in Table 1 . The soon to be flight proven PHI instrument on board Solar Orbiter will provide a good base for developing the PIM instrument. The changes of design would mostly be focused on the single telescope adaption to the new orbit and the extension of the field of view. A higher observation cadence, although not mandatory, could be very valuable to our understanding of erupting ARs.

EUV Active Region Imager (EUVARI) - The EUV imager will provide simultaneous measurements of the full solar disk in two different wavelengths on each spacecraft. Since an instrument with these properties and suitable mass does not exist, technological developments will be needed to produce an instrument that fulfils our requirements. This new instrument will consist of two telescopes. One of these will observe the lower corona in the $17.1 \mathrm{~nm}$ wavelength, and the other will be able to switch between 9.4 and $21.1 \mathrm{~nm}$ using a filter wheel. These wavelength bands cover the footprints of the loops and the loops themselves. They can also be used to detect solar flares (see Sect. 6.2 for an operational use of those filters). To provide more flexibility, to simplify the design and to provide some redundancy, both telescopes will be equipped with filter wheels covering each of the three wavelengths.

Significant heritage is available for such an instrument: the SDO, STEREO and Solar Orbiter missions have all flown, or will fly, instruments with some of the characteristics matching our requirements. For instance, the EUV imager on Solar Orbiter will have two telescopes with a combined mass of $23.5 \mathrm{~kg}$, but the filters and fields of view must be changed to suit an orbit at $1 \mathrm{AU}$. However, the development and testing of this instrument will be the greatest design challenge of the mission (see Sect. 4.3 for details). The simultaneous stereoscopic 


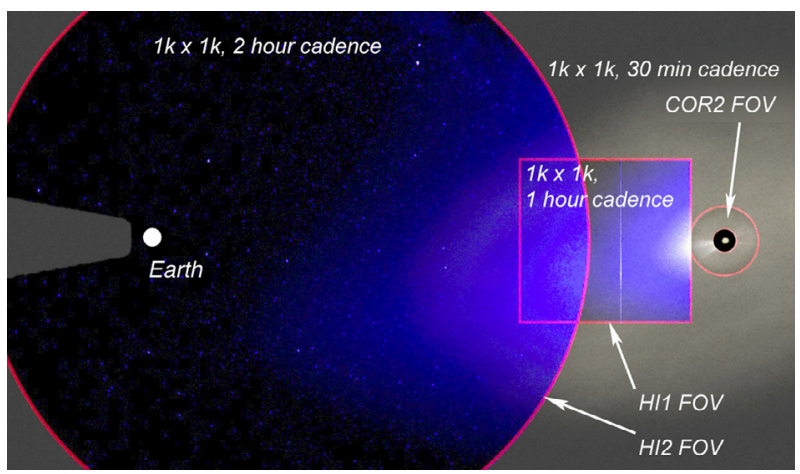

Fig. 3. The combined field of view of the OSCAR coronal and heliospheric imagers, adapted from the STEREO gallery. The Earth, not to scale, is labelled for reference.

observations - at high resolution - of the photospheric magnetic field and the upper coronal structure in ARs will be the key to characterise in detail the trigger of CMEs.

Coronagraph (COR) - The coronagraph (COR) is an externally occulted Lyot coronagraph, which will be capable of imaging the electron density of the solar corona through observing the polarised brightness of Thomson scattered light. To reduce instrument development costs, we can rely on the COR2 instrument flying on the STEREO spacecraft. COR will provide data on early CME propagation between 2 and $15 \mathrm{R}_{\odot}$ for first hazard assessments. The instrument is designed to observe moderately fast CMEs moving with speeds of about $750 \mathrm{~km} / \mathrm{s}$, which covers the average initial CME speeds as seen in LASCO data (Manoharan \& Mujiber Rahman 2011). Like its heritage instrument, it will produce a three-polarisation image sequence in visible light. This will enable us to observe the polarised brightness of the coronal plasma, which can be directly related to the distribution of electrons (using, e.g., the solar rotational tomography method, see Frazin \& Kamalabadi 2005). To observe the average CME event, short exposure times of $<4 \mathrm{~s}$ are necessary to avoid pixel smear. Faster events can also be resolved by binning pixels (Howard et al. 2008).

Heliospheric Imagers (HI) - Given the success of the stereoscopic reconstruction with STEREO's Heliospheric Imager (HI; see, e.g., Lugaz et al. 2012), the same instrument is also planned to be reused aboard the OSCAR spacecraft. The HI will take over CME observation once they are out of the coronagraph's field of view, following CMEs from 12 to $277 \mathrm{R}_{\odot}$, corresponding to an $85^{\circ}$ field of view on the Sun-Earth line. It consists of the two cameras HI-1 and HI-2 to cover the decreasing intensity of visible Thomson scattered light during the CME's propagation. The opening angles also correspond to typical average CME widths which have been found to range between $47^{\circ}$ and $61^{\circ}$ from solar minimum to maximum (Yashiro et al. 2004). Due to the different intensities, the exposure times vary between HI-1 and HI-2. Exposure times of 12-20 s with typically 150 exposures per image for HI-1 and 60-90 s exposure time with typically 100 exposures per image allow a nominal image cadence of 60 and $120 \mathrm{~min}$ for this instrument (Howard et al. 2008). Using the COR and HI the volume in which CMEs can be viewed then reaches from $2 \mathrm{R}_{\odot}$ to beyond $1 \mathrm{AU}$, as seen in Figure 3 .

\subsection{In-situ instrumentation}

The in-situ instrumentation aboard each of the OSCAR spacecraft consists of two identical magnetometers and two different 
particle instruments, one measuring the solar wind particles, the other one the shock-accelerated high energy electrons, low energy protons and alpha particles. The overall mass of the in-situ instrumentation package is estimated to be $29.4 \mathrm{~kg}$, the power consumption will be $17.0 \mathrm{~W}$.

Solar Wind Particle Monitor (SWPM) - The SWPM will measure the 1D velocity distribution functions (VDFs) of solar wind protons, alpha particles and the more abundant charge states of certain heavier elements $(\mathrm{C}, \mathrm{O}, \mathrm{Si}, \mathrm{Fe})$ in the solar wind as well as the 3D-VDF of solar wind electrons. Due to the large differences between the expected particle fluxes, the proton/alpha measurement and the heavy ion measurement will be done separately with two different sensors SWPM-AP and SWPM-HI both mounted on the spacecraft body looking towards the Sun. SWPM-AP will measure protons and alphas in the velocity range between $180 \mathrm{~km} / \mathrm{s}$ and $2100 \mathrm{~km} / \mathrm{s}$. Thus the velocity range is extended compared to regular solar wind speeds in order to measure not only CIRs but even high CME bulk speeds.This design is directly taken from its heritage instrument ACE/SWEPAM which has an energy-per-nucleon range between $260 \mathrm{eV} /$ nuc and $36 \mathrm{keV} /$ nuc and a relative energy-per-nucleon resolution of 5\% (McComas et al. 1998). SWPM-HI will be able to measure the charge states of carbon, oxygen, silicon and iron with up to $100 \mathrm{kev} / \mathrm{nuc}$ in order to determine locally the stream interfaces in CIRs and transient CMEs. The third sensor SWPM-E finally will measure the solar wind electrons. As heritage instruments for SWPM-E we propose STEREO/SWEA (Sauvaud et al. 2008), which can determine $3 \mathrm{D}$ velocity distributions of electrons in the energy range between $1 \mathrm{eV}$ and $3 \mathrm{keV}$. As its heritage instrument SWPM-E will be mounted on the spacecraft boom.

Energetic Particle Monitor (EPM) - The EPM instrument consists of two identical sensor pairs which will both measure low energetic proton and alpha particles covering ion energies from $46 \mathrm{keV}$ up to $4.8 \mathrm{MeV}$, and electron energies between $40 \mathrm{keV}$ and $350 \mathrm{keV}$. One sensor pair EPM-1 will be tilted $\left(-45^{\circ}\right)$ in longitude with respect to the sun-spacecraft line to capture the high energetic electrons that are expected to propagate along the spiraling interplanetary magnetic field. The second sensor pair EPM-2 will point $180^{\circ}$ away from the first sensor. This design, implying a rough spatial resolution within the ecliptic, will enable us to (i) identify anisotropic electron events, which are the relevant ones in the context of CME shock accelerated particles (Simnett et al. 2002) and (ii) distinguish between low energetic ions accelerated inside or beyond $1 \mathrm{AU}$. The heritage instrument is ACE/EPAM (Gold et al. 1998), but note that the sensor mounting would have to be adapted to the three-axis-stabilised OSCAR spacecraft as described above.

Magnetometers (MAG) - The MAG instrument consists of two identical fluxgate magnetometers. Each of them will measure all three components of the local interplanetary magnetic field vector in the energy range $\pm 200 \mathrm{nT}$ with an absolute accuracy of $0.1 \mathrm{nT}$ and an operational time resolution of 1 minute for space weather forecasting. Subsecond time-resolved data will be available for scientific studies of the magnetic field, such as wave and turbulence phenomena in the solar wind. The magnetometers will be synchronised and both mounted on the spacecraft boom at a distance of $1.0 \mathrm{~m}$ and $2.25 \mathrm{~m}$ from the spacecraft body, respectively. This will allow us to reconstruct the magnetic background of the spacecraft itself. The Solar Orbiter MAG (Carr et al. 2007) will serve as heritage instrument for the MAG instrument. The combination of MAG and SWPM data will provide the necessary data for a reliable CIRs forecasts in real-time.

\subsection{Critical technology requirements}

Technically a large proportion of the risk relies on the successful implementation of the EUV Active Region Imager. To minimise it the EUVARI telescope will be based on three already developed EUV imagers, which by the estimated time of launch will each be flight-proven. Since the data produced by this instrument are critical for mission success, redundancy in certain crucial wavelengths imaging will be built into the design in case of damage during launch or flight.

Another large proportion of the risk to our mission lies in the requirement to successfully implement major changes in the PIM instrument compared to its heritage instrument PHI: doubling the $16.8^{\prime}$ field of view of PHI - to ensure a full-disk field of view while maintaining the high spatial and time resolution - is the critical challenge for the successful realisation of this instrument. However, abandoning the two-telescope design of PHI should save weight and make room for PIMspecific changes, therefore keeping it compact and lightweight.

\section{Spacecraft design}

We give now a brief tour of the design of the OSCAR twin spacecraft. The system architecture and satellite design are shown in Figure 4. The OSCAR spacecraft are three-axisstabilised spacecraft that actively point the imaging instruments towards the Sun. The Attitude Determination and Control System (ADCS) computes the attitude and if necessary utilises reaction wheels and lateral thrusters to alter the orientation. Solar panels are utilised to harvest energy which is processed towards the Power Control and Distribution Units (PCDUs) and stored in the batteries. The communication subsystem consists of two redundant X-band transceivers connected to a high gain antenna and two low gain antennas.

\subsection{Mass budget}

Table 3 summarises the mass budget for each OSCAR spacecraft. The estimated mass for each spacecraft is $580.5 \mathrm{~kg}$ including - on top of subsystem margins - an additional margin of $20 \%$. If one Soyuz launcher is used for both spacecraft (see Sect. 6.1), an unused mass of $28.2 \mathrm{~kg}$ for each spacecraft provides us a comfortable margin.

For structure, thermal control, onboard computer (OBC) and data handling $(\mathrm{DH})$ a margin of $10 \%$ is provisioned. A margin of $5 \%$ is assumed for the subsystems ADCS and EPS thanks to space heritage. The margins of scientific payload and telemetry, tracking and command (TT\&C) result from the single margins of the subsystems components, based on the standard ESA margin policy. The weight of harness is estimated to $5 \%$ of the net spacecraft mass.

\subsection{Power budget}

The power system consists of three main modules: the primary module, the secondary module and the PCDUs.

The primary module covers the main power harvesting in order to operate the satellite. The triple junction solar cells with a GaInP2/GaAs/Ge composition (produced, e.g., by Spectrolab), 

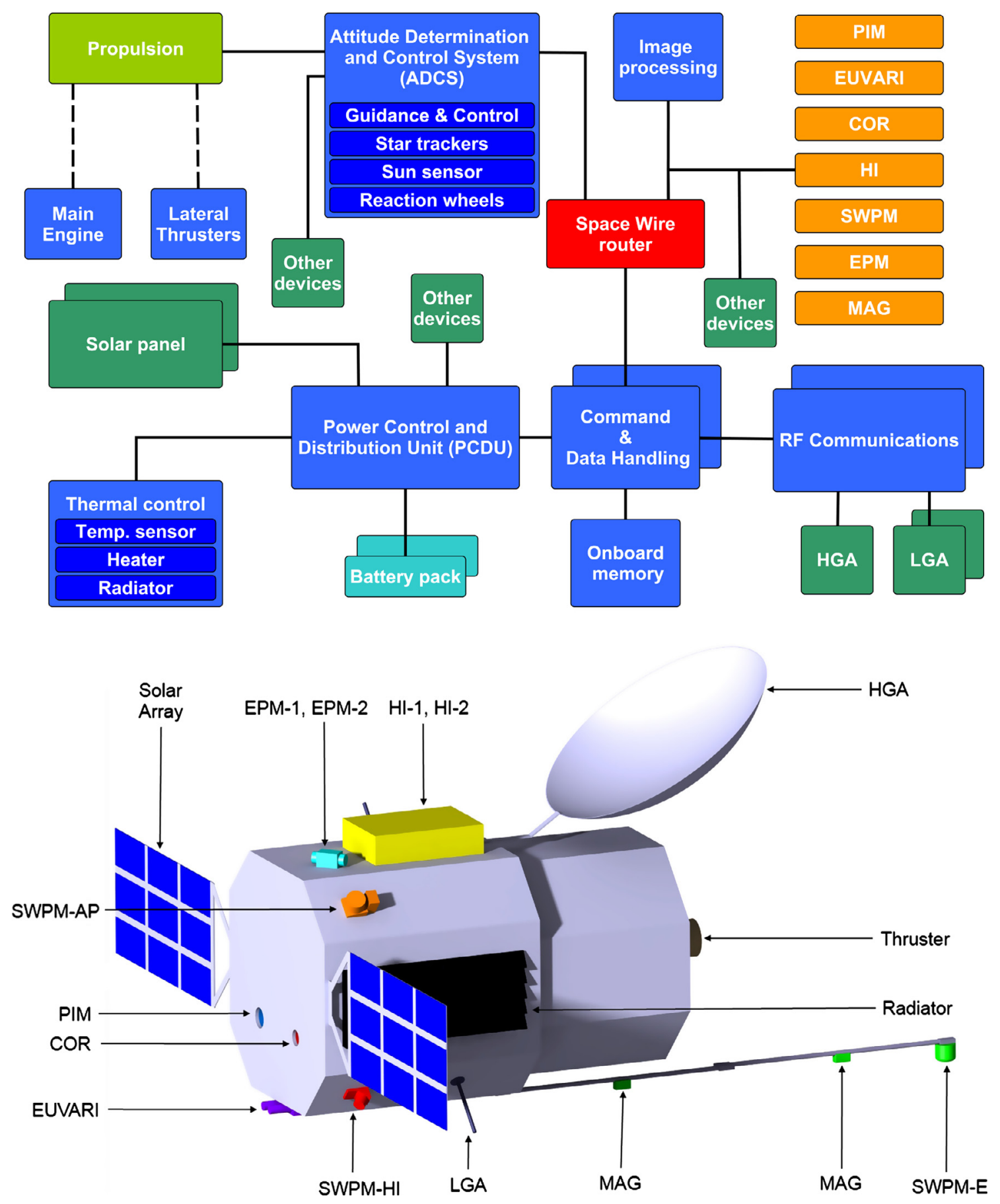

Fig. 4. System architecture and spacecraft design. The estimated dimensions of the (folded) spacecraft are $1.6 \times 1.6 \times 2.7 \mathrm{~m}$.

which are designed for space missions, could be used. Each satellite would embark a solar panel area of $2.5 \mathrm{~m}^{2}$ featuring an efficiency of $29.5 \%$. When the solar panels are exposed to direct sunlight at a distance of $1 \mathrm{AU}$, the maximum power conversion achievable is calculated to be $905.1 \mathrm{~W}$ with the assumption of surface temperature of $28{ }^{\circ} \mathrm{C}$. The radiation degradation of the solar cells after 7 years of operation time (end of nominal mission) is expected to be approximately $10 \%$.

The secondary module consists of the backup power stored in rechargeable lithium ion batteries. For a long lifetime, the batteries are discharged to $50 \%$ of the full capacity and charged to $90 \%$ of the full capacity. The total energy capacity of the battery pack is calculated to be $880 \mathrm{Wh}$ with the assumption that the batteries operate at $20{ }^{\circ} \mathrm{C}$.
The distribution of the power is handled by two low-power PCDUs from Thales Alenia Space which manage the batteries and the maximum peak power tracking for power harvesting. Each PCDU is able to deliver up to $330 \mathrm{~W}$.

Table 4 summarises the power budget for each spacecraft of the proposed mission OSCAR. The margin for the OBC\&DH including the solid state recorder (SSR) is assumed to be $10 \%$. For other subsystems which still require more modifications a margin of $20 \%$ is assumed. This applies to TT\&C, EPS or propulsion system. The margins of scientific payload and ADCS result from single margins of the components the subsystems comprise of based on the standard ESA margin policy. The estimated power for each spacecraft is $855.4 \mathrm{~W}$ including on top of subsystem margins an additional margin 
A. Strugarek et al.: Observatories of the Solar Corona and Active Regions (OSCAR)

Table 3. Dry mass budget. The margins are averages based on the standard ESA margin policy.

\begin{tabular}{lccr}
\hline \hline Subsystem & Nominal mass $(\mathrm{kg})$ & Margin $(\%)$ & Sum $(\mathrm{kg})$ \\
\hline Structure & 149.0 & 10.0 & 163.9 \\
Payload* & 110.5 & 10.1 & 121.6 \\
TT\&C & 23.1 & 5.6 & 24.4 \\
ADCS & 32.1 & 5.0 & 33.7 \\
OBC\&DH & 25.0 & 5.0 & 27.5 \\
EPS & 18.1 & 5.0 & 19.0 \\
Thermal & 15.2 & 10.0 & 16.7 \\
Propulsion & 53.0 & 5.0 & 55.7 \\
Harness & 21.4 & - & 21.4 \\
\hline Sum & - & - & 483.8 \\
Margin & - & 20.0 & 96.8 \\
Total & - & - & 580.5 \\
Maximum & - & - & 608.8 \\
Unused & - & - & 28.2 \\
\hline
\end{tabular}

*A boom of $3.2 \mathrm{~kg}$ has been added to the payload mass estimate (see Table 2).

Table 4. Power budget. The margins are averaged from the standard ESA margin policy.

\begin{tabular}{lccc}
\hline \hline Subsystem & Nominal power $(\mathrm{W})$ & Margin $(\%)$ & Sum $(\mathrm{W})$ \\
\hline Payload & 91.8 & 12.3 & 103.1 \\
TT\&C & 200.0 & 20.0 & 240.0 \\
ADCS & 205.0 & 7.9 & 221.3 \\
OBC\&DH & 75.0 & 10.0 & 82.5 \\
Propulsion & 55.0 & 20.0 & 66.0 \\
\hline Sum & - & - & 712.8 \\
Margin & - & 20.0 & 142.6 \\
Total & - & - & 855.4 \\
Maximum & - & - & 905.1 \\
Unused & - & - & 49.8 \\
\hline
\end{tabular}

of $20 \%$, which provides us with an unused power of $49.8 \mathrm{~W}$ per spacecraft.

\subsection{On board computer, data handling and telemetry}

The data on board the satellite will be handled by an On Board Computer (OBC) of the type OSCAR (coincidentally) manufactured by EADS Astrium. The OBC utilises the LEON3 core and provides up to $40 \mathrm{MIPS}$ at $48 \mathrm{MHz}$ core frequency. With $256 \mathrm{MB}$ of RAM and $512 \mathrm{MB}$ of exchange memory the computer meets our requirements. Not only the telemetry data and command handling but also the execution of the ADCS algorithms and time synchronization can be performed on the $\mathrm{OBC}$. The processing and analysis of acquired images is dedicated to a separate image processing unit.

The images produced by the scientific instruments will generate a large amount of data (see Sect. 6.2), some of which will be required to be stored on board before being downloaded on Earth. A flight-proven EADS Astrium SSR based on the flash technology could be utilised to ensure a storage capacity of 20 Tbits $(\sim 2.5 \mathrm{~TB})$. The power consumption is estimated to be $60 \mathrm{~W}$ and its mass to be $20 \mathrm{~kg}$ based on a realistic increase of performances of SSR in the coming years. These specifications are included in the corresponding budgets (Tables 3 and 4) in the OBC\&DH entry.
Table 5. Known thermal requirements for spacecraft and payload components.

\begin{tabular}{lcc}
\hline \hline Component & $\begin{array}{c}\text { Operational } \\
\text { temperature }\left[{ }^{\circ} \mathrm{C}\right]\end{array}$ & $\begin{array}{c}\text { Survival } \\
\text { temperature }\left[{ }^{\circ} \mathrm{C}\right]\end{array}$ \\
\hline $\begin{array}{l}\text { Batteries } \\
\text { Reaction }\end{array}$ & -5 to 50 & -15 to 60 \\
wheels & -5 to 50 & -20 to 60 \\
Sun sensor & & \\
Star trackers & & -40 to 90 \\
OBC\&DH & -80 to 95 & -40 to 70 \\
Antenna & -30 to 60 & -40 to 75 \\
Gimbals & -20 to 60 & -50 to 90 \\
Antennas & -40 to 80 & -120 to 120 \\
Solar panels & -100 to 100 & -200 to 130 \\
MAG $^{\mathrm{a}}$ & -150 to 120 & -100 to 100 \\
SWPM $^{\mathrm{a}, \mathrm{d}}$ & -100 to 100 & -30 to 60 \\
EPM $^{\mathrm{a}}$ & -25 to 50 & -30 to 60 \\
$\mathrm{COR}^{\mathrm{e}}$ & -25 to 50 & -20 to 55 \\
$\mathrm{HI}^{\mathrm{e}}$ & 0 to 40 & -60 to 60 \\
\hline
\end{tabular}

${ }^{\mathrm{a}}$ Wertz \& Larson (2003); ${ }^{\mathrm{b}}$ Carr et al. (2007); ${ }^{\mathrm{c}} \mathrm{McComas}$ et al. (1998); ${ }^{\mathrm{d}}$ Sauvaud et al. (2008); ${ }^{\mathrm{e}}$ Howard et al. (2008).

The data will be downloaded to the ground station (or ground station network) periodically. Because of the large distance between the spacecraft and the Earth, the telemetry design is particularly critical for the feasibility of OSCAR. In order to provide sufficient downlink budget, X-band communication is utilised. Two redundant transceivers with the output power of $200 \mathrm{~W}$ each feeding a $1.7 \mathrm{~m}$-diameter parabola antenna will ensure a downlink data rate of $1.4 \mathrm{Mbps}$ if the ESA ESTRACK network is used, and $260 \mathrm{kbps}$ if smaller $15 \mathrm{~m}$ ground station antennas are utilised. The total daily data budget would then be $2.35 \mathrm{~GB}$ and $218 \mathrm{MB}$, respectively. In both cases the signal margin of $3 \mathrm{~dB}$ is maintained in order to guarantee proper operation. We demonstrate in Section 6.2 how such a reasonable telemetry budget is able to meet the scientific requirements of the mission, and propose alternative budgets depending on the ground stations availability.

\subsection{Thermal control subsystem}

The role of the thermal control subsystem is to maintain all spacecraft and payload components within their required temperature limits during the mission. Table 5 shows the thermal requirements for each component of the spacecraft. The PIM instrument inherits its property from the future PHI instrument that will fly on Solar Orbiter. Its operational and survival temperature ranges are still not exactly known today. Similarly, the exact EUVARI instrument does not exist yet (see Sect. 4.3), and its operational temperature ranges can only be speculated. In addition, those two instruments would include their own thermal system. For these reasons, we chose not to consider them in the following preliminary analysis.

A thermal analysis is generally needed to define an adequate radiator area to accommodate the maximum operational power during the hottest and coldest operational environment, without exceeding the allowed temperatures of $0{ }^{\circ} \mathrm{C}$ and $30{ }^{\circ} \mathrm{C}$ (see Table 5).

As a first approximation it is possible to assume an isothermal and spherical spacecraft with a radius equal to the maximum dimension of the longest subsystem $(\sim 1 \mathrm{~m})$ located at $1 \mathrm{AU}$ from Sun and 0.59 AU from Earth. The satellites will 
be heated continuously by the direct solar radiation during the whole mission.

The temperature of each spacecraft depends on the balance between its absorbed, internally diffused and externally radiated thermal power. The internal power dissipation varies from 99.3 W (safe mode) to $858.2 \mathrm{~W}$ (nominal mode). By considering a spacecraft emittance of 0.8 and an absorptivity of the solar radiation of 0.6 , one finds equilibrium temperatures of $-3.85{ }^{\circ} \mathrm{C}$ and $48.99{ }^{\circ} \mathrm{C}$, respectively. A radiator (with an emittance of 0.9 ) of $3.42 \mathrm{~m}^{2}$ would be required to accommodate the internal dissipation power in normal operations. This unrealistically large radiator area promotes a more detailed thermal analysis that could follow two paths. Because the OSCAR spacecraft will conserve their orientation with respect to the Sun during the mission, heat shields could be designed on the Sun-facing part of the spacecraft to lower the internal temperature. In a perfect-shield case, a radiator of $1.98 \mathrm{~m}^{2}$ would suffice to maintain the internal temperature in the operational range. Alternatively, an active internal cooling system could also be designed to accommodate the internal power dissipation. Finally, the spacecraft temperature in safe mode is very close to the survival and operational limits, henceforth a passive thermal control could be designed to maintain an acceptable temperature in this case. This basic thermal analysis shows that the design of OSCAR is a priori feasible.

\section{Mission design}

After the description of the instruments and spacecraft design of OSCAR, we now give insights into the mission design. We detail the possible orbits of the spacecraft and the operational phases in Section 6.1. We then propose operational modes for the scientific and forecast data (Sect. 6.2). We finally give an overview of a possible ground segment design (Sect. 6.3).

\subsection{Planned orbit and operational phases}

The OSCAR spacecraft will be inserted into a heliocentric orbit at a distance of $1 \mathrm{AU}$ from the Sun with one spacecraft leading the Earth and other trailing behind with a separation angle of $68 \pm 3^{\circ}$ (see Fig. 2). This configuration will allow an optimum observation of the Sun surface to study CMEs and coronal loops, and the optimal acquisition of binocular high-resolution images as explained in Section 3.2. Additionally, observation of the CME propagation along the way to the Earth will also be possible.

Five operational phases have been identified for the entire mission: (i) the launch, (ii) a two-year spacecraft drift period, (iii) 5 years of nominal mission time, (iv) a possible mission extension and (v) potential deorbiting.

The total launch mass, i.e. the sum of dry mass $(\sim 1161 \mathrm{~kg}$, see Table 3$)$, the propellant mass $(\sim 575 \mathrm{~kg})$ and the mass of the payload adapters $(\sim 270 \mathrm{~kg})$, is approximately $2006 \mathrm{~kg}$. A Soyuz rocket, which is capable of delivering $2200 \mathrm{~kg}$ to an Earth escape trajectory, has therefore been selected for the mission (the two OSCAR spacecraft also fit in the Soyuz rocket fairing, as shown in Fig. 5). After escaping the Earth gravity, the two spacecraft will each perform a $0.47 \mathrm{~km} / \mathrm{s}$ delta-v manoeuvre in opposite directions i.e. Oscar 1 (leading Earth, see Fig. 2) will perform a retrograde burn and Oscar 2 (trailing Earth) a prograde burn with respect to their heliocentric orbit. This marks the start of a 2-year drift phase for both the
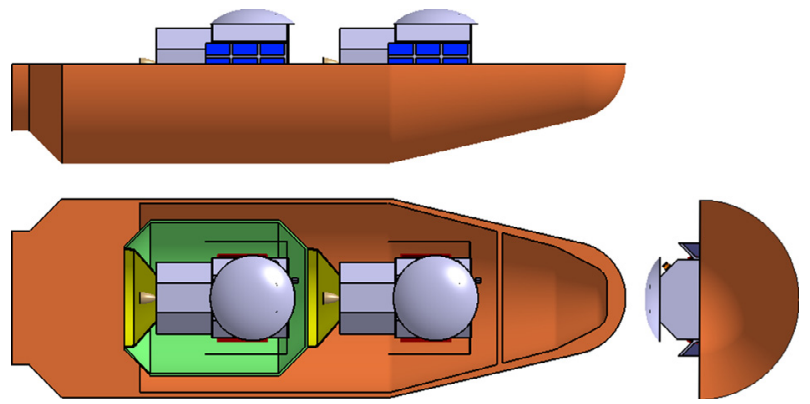

Fig. 5. The two OSCAR spacecraft stacked in a Soyuz rocket fairing.

spacecraft. At the end of this phase each spacecraft will have reached its final target position relative to Earth and will require another $0.47 \mathrm{~km} / \mathrm{s}$ delta-v manoeuvre to stop the drift in order to maintain the final constellation. The distance between the spacecraft and Earth will be about 0.59 AU.

The launch will take approximately $30 \mathrm{~min}$ for the spacecraft to reach the Earth escape trajectory. Once they reach this point, their propulsion system will be activated and the drift phase will begin. After about 8 months the angle between the Sun and both spacecraft will reach $22^{\circ}$ which is the minimum requirement for the spacecraft to start performing observations to achieve part of our mission objectives (Sect. 3.2). Additionally, at this stage we can start evaluating and if necessary optimise the data handling and propagation of data from the ground stations to various data centres that are mentioned in Section 6.3. The scheduled science operation phase is 5 years. However, since we have considered generous margin for the propellant and the power budgets, an extension of the mission duration is optional. After the end of mission both spacecraft will be transferred to a disposal orbit around the Sun with the major semi-axis length of $0.99 \mathrm{AU}$.

\subsection{Operational mode and ground segment}

\subsubsection{CME trigger data}

Since our primary objective is to study the trigger mechanism(s) of CMEs in high detail, and we can only transmit a limited amount of data to ground stations due to the satellites distance from Earth, onboard autonomy is clearly required. Instead of sending data from all instruments at full cadence and full resolution, our satellites will perform onboard CME trigger event detection using one of the EUV telescopes. We will use a dedicated image processing unit as well as customisable CME trigger event detection software. The following operation mode (summarised in Fig. 6) shall enable OSCAR to fulfil its primary objective in spite of the telemetry limitations.

Both EUVARI and PIM telescopes shall continuously buffer images at full resolution and their fastest cadence (a). The buffering shall be synchronised using ground stations on both satellites taking into account their distance to the Sun. One EUV telescope will record images in $17.1 \mathrm{~nm}$, while the other can switch between the $9.4 \mathrm{~nm}$ and the $21.1 \mathrm{~nm}$ channel. The trigger detection shall be based on the detection of strong flares in the $9.4 \mathrm{~nm}$ channel. Whenever a strong flare is detected (b.1) the satellite will change the $9.4 \mathrm{~nm}$ filter to $21.1 \mathrm{~nm}$ for the next hour (c). This ensures that we always have $17.1 \mathrm{~nm}$ images available, and most of the time right after an event is detected also $21.1 \mathrm{~nm}$ images. After $1 \mathrm{~h}$ the satellite 


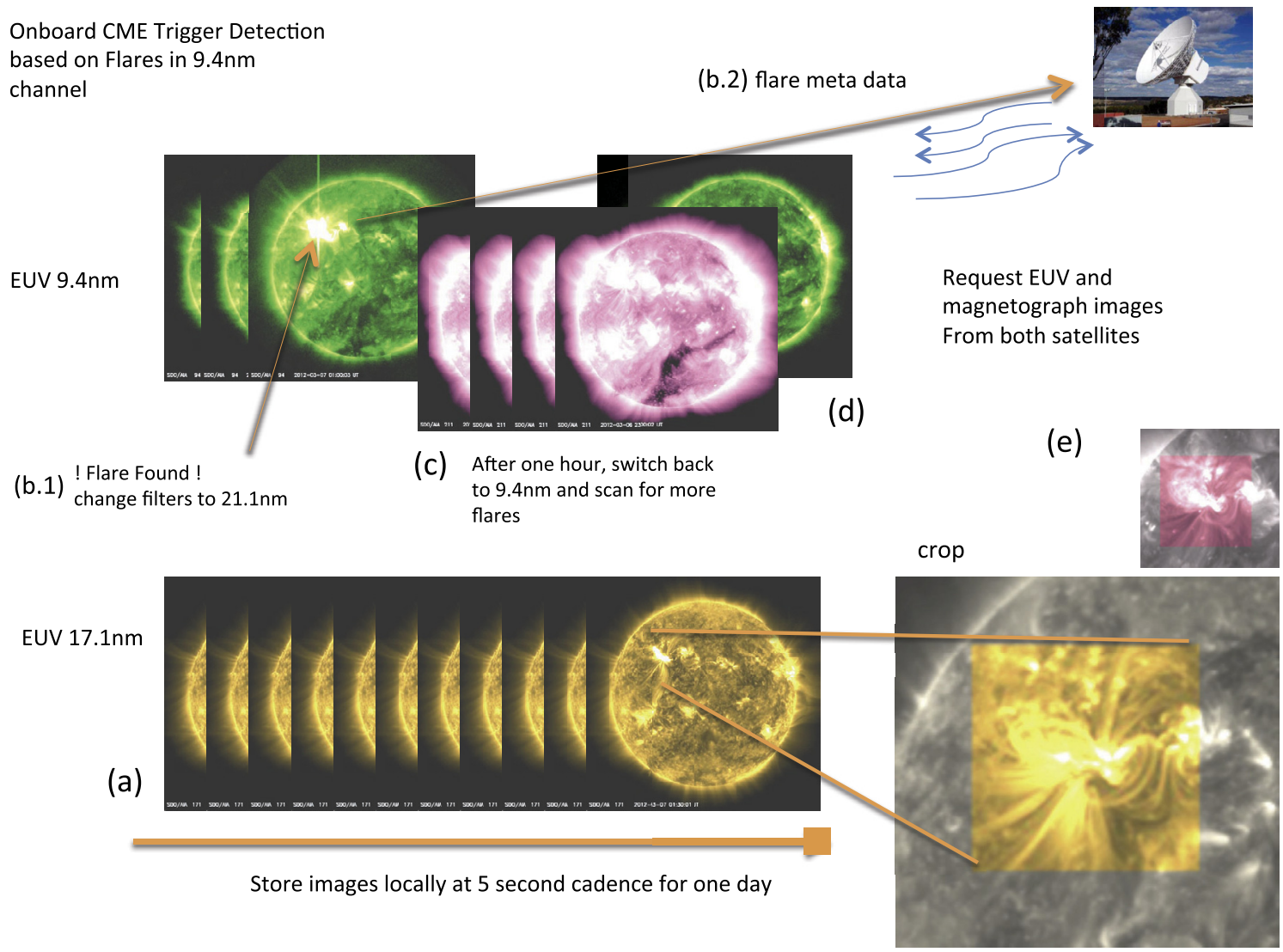

Fig. 6. OSCAR operational mode for the CME trigger data. See text for details on this particular operational mode.

Table 6. Onboard mass storage and expected telemetry: CME Trigger (for one spacecraft).

\begin{tabular}{lccccc}
\hline \hline Instrument & Lossless & Resolution [px] & Cadence [s] & Onboard storage [MB/day] & Telemetry [MB/day] \\
\hline EUVARI & $y$ & $4 \mathrm{k} \times 4 \mathrm{k}$ & 5 & 552,960 & 307 \\
& $n$ & $4 \mathrm{k} \times 4 \mathrm{k}$ & 3600 & & 554 \\
PIM & $y$ & $800 \times 800$ & 5 & 30,720 & 154 \\
& $y$ & $2 \mathrm{k} \times 2 \mathrm{k}$ & 45 & & 31 \\
Total & $y$ & $400 \times 400$ & 4600 & 583,680 & 1045 \\
\hline
\end{tabular}

will switch back to the $9.4 \mathrm{~nm}$ channel to continue the online flare detection $(\mathrm{d})$.

The output of the event detection is an estimate of the class of the flare, as well as the location. This meta-data will be sent back to a ground station (b.2), where, based on the trigger detection meta-data from both satellites and possibly other sources, it is decided which data from both the EUVARI and PIM instruments to request from both satellites (e). It is also possible to request any of the other buffered data, given external trigger detection using third-party data. Furthermore, if simultaneous CMEs were to be triggered, the data could still be retrieved since the EUV telescopes are continuously imaging the full-disk of the Sun.

Other possibilities for online CME trigger event detection can be based on dimmings and EUV waves, instead of on strong flares. Both dimmings and EUV waves are strongly related to the onset of CMEs (Zhukov \& Auchere 2004). An advantage over the flare detector operating on $9.4 \mathrm{~nm}$ images would be that both EUV telescopes can continuously record in the wavelengths that are best suited for coronal loop imaging (17.1 and $21.1 \mathrm{~nm}$ ), also for the minutes leading up to CME trigger event. Both EUV waves and dimming detectors as well as flare detectors are currently being developed at the Royal Observatory of Belgium as part of the FP7 project AFFECTS. These can be adapted for near-real time operation on satellites.

Instead of requesting full-resolution data, cropped images will be downloaded for a time period spanning from $10 \mathrm{~min}$ before the event until $60 \mathrm{~min}$ after the event was detected. This ensures the total telemetry, given on average $200 \mathrm{M} 1$ or stronger flares per year (Tang \& Le 2005), does not exceed $1235 \mathrm{MB}$ per day for each satellite (see the budget including regular and science data in Table 6). To retrieve this data we make use of the Deep Space Antennas (DSA) of the ESA's ESTRACK network, using one timeslot of $8 \mathrm{~h}$ each day per satellite. The DSA consists of three $35 \mathrm{~m}$-diameter antennas and is designed to ensure constant availability for spacecraft distant from Earth. It provides the highest telemetry rate 


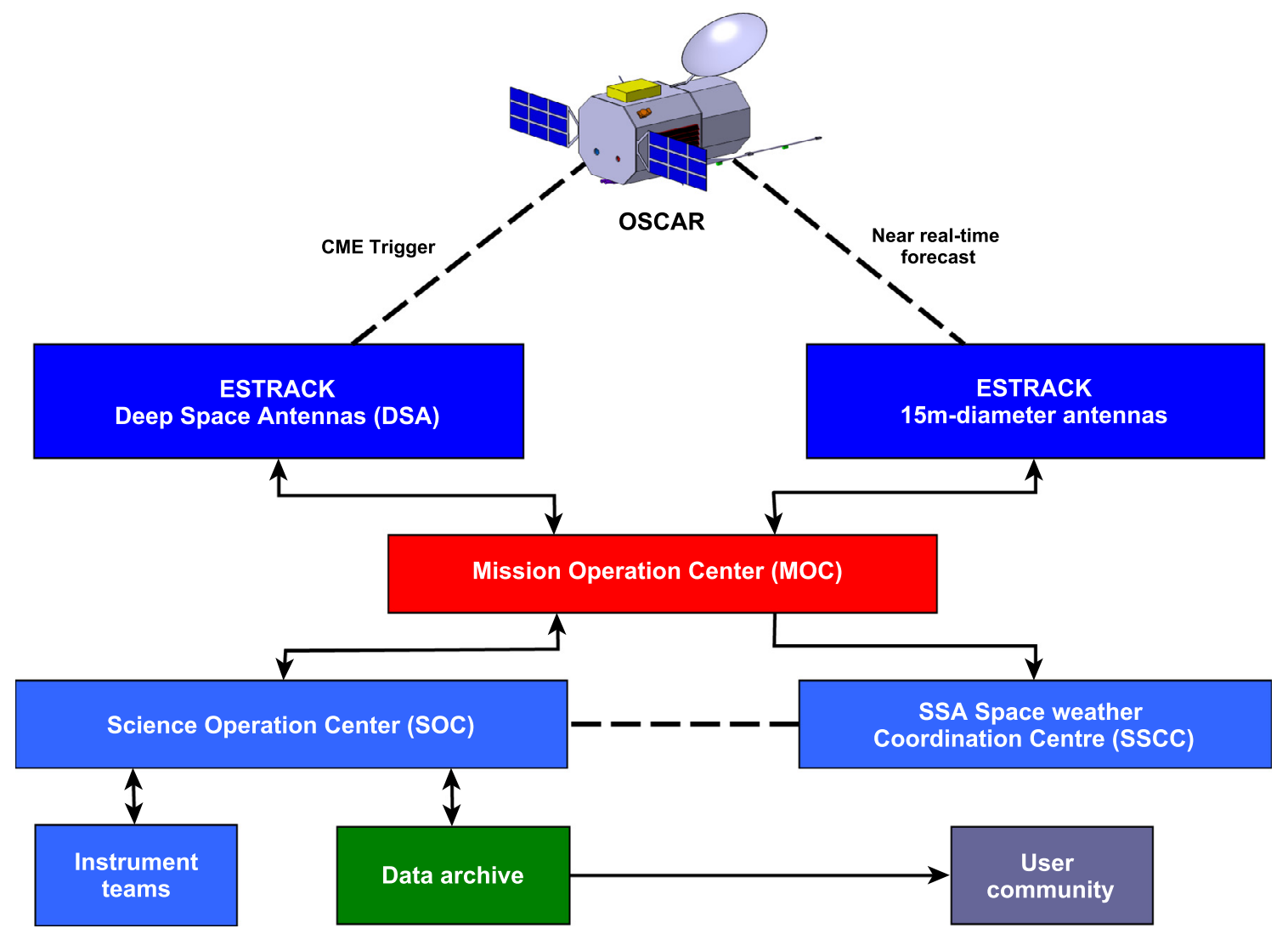

Fig. 7. OSCAR ground segment design.

available on Earth for our mission. The onboard storage can be viewed as a buffer for the present discussion, containing events of potential interest from which only a portion will be downloaded. The large onboard storage (Sect. 5.3) enables a storage of 4 days of the whole science data. Depending on the available timeslots of the DSA, more data could be downloaded, other operational modes could be proposed, and calls for allocation time could then be launched. The possibility of using the smaller 15 m-diameter antennas of the ESTRACK network (in total six antennas) could also be considered for those imagers as long as this does not affect the downlink time required for the forecasting aspect of the OSCAR mission (see next section). Nevertheless, the $15 \mathrm{~m}$-diameter antennas would not support the large amount of the stereoscopic observations by itself and should only be used as complementary antennas.

\subsubsection{Real-time forecasting}

For near-real time forecasting we rely on the availability of $15 \mathrm{~m}$ telescopes that receive data from both satellites every $6 \mathrm{~h}$. The total telemetry for near-real time forecasting is estimated to be $47 \mathrm{MB}$ every $6 \mathrm{~h}$ per satellite. This includes telemetry for the coronograph (36 MB), the HI instruments $(9 \mathrm{MB})$ and the in-situ measurements provided by the Particle monitor and Magnetometer (2 MB). This amount of data can be transferred in less than $1 \mathrm{~h}$. The $15 \mathrm{~m}$ antennas of the ESTRACK network could in principle be used for such telemetry. While this demonstrates the feasibility of OSCAR's forecasting objectives, it could also be considered to develop dedicated infrastructures to ensure a more reliable forecasting system.

\subsection{Ground segment}

The operational modes also require a specific design for the mission ground segment. A schematic of our ground segment operation is given in Figure 7.

Our design involves the communication with the ESA's ESTRACK network wherein the DSA is used for CME trigger study and the $15 \mathrm{~m}$ diameter antennas for the forecasting data. Our Mission Operation Center (MOC) would provide an interface between the two antenna networks and the science operation and space weather centres. It would also interact with the two satellites for the data downloading requests.

We would of course aggregate a scientific community around our CME trigger study. Specific partner research institutes would be involved in the analysis and use of the data to achieve our first mission objective. The forecast data would be directly interfaced with e.g., the SSA Space Weather Coordination Centre (SSCC) through our MOC. A constant link between SSCC and the OSCAR SOC would enable a good use of our forecast data. The forecast itself will be either provided by the SOC or by SSCC, depending on the available manpower. Subsequently, SSCC would be in charge of releasing the forecast and alerts obtained from our data. Additionally our forecast data may be combined with other spacecraft data to provide a better insight into the 3D structure and temporal evolution of CMEs and CIRs at $1 \mathrm{AU}$.

\section{Cost analysis and descoping option}

We give here rough cost estimates of the OSCAR mission in the eventuality of a launch at the horizon 2022-2025. 
Thanks to the compact design of OSCAR (Sect. 5), one Soyuz launcher can be used for the two satellites. It has the advantages of a (relatively) low cost and high reliability. A nearequatorial launch location is compatible with the orbit design (Sect. 6.1) and costs $60 \mathrm{M} €$ from Kourou. Predictably weight, power and performance are the main cost drivers. The largest cost in the mission is for the platform (255 M€) which represents $57 \%$ of the cost, for which orbit and attitude control and data management represent a large proportion of the platform budget. The payload cost is also considerable, estimated at $170 \mathrm{M} €$ (estimate of $1 \mathrm{M} € / \mathrm{kg}$, see Wertz \& Larson 2003). The development cost of the improved EUV imager is estimated at $5 \mathrm{M} €$ by itself. Adding the forecast (100 M€) and science $(60 \mathrm{M} €)$ operations costs, the total cost for the two satellites mission is estimated around $650 \mathrm{M€}$.

Should it be necessary to reduce the scope of the mission, particularly to reduce the overall cost, the following option has been investigated. Since both spacecraft have identical instrumentation, a possibility would be launch only one of them. For this to retain the objective of loop reconstruction, this spacecraft would need an orbit relocated at L5 and would rely on additional image data in the equivalent wavelengths and cadences from L1 or Earth, using already flying satellites. If this could be provided by other partners the primary objective could be fulfiled, while investigating a different portion of the Sun's surface. However, the matching of suitable data would be a significant challenge, and data would likely need to be processed and interpolated to match in time with the mission data. In addition, the data rate would be expected to drop by $64 \%$ compared to the nominal mission plan due to the additional spacecraft distance. Although feasible, it clearly appears that this solution would seriously threaten the mission while saving only a sixth of its cost.

\section{Conclusions}

We reported a first study for an innovative space weather mission concept, OSCAR. We presented the scientific basis for a twin spacecraft mission, leading and trailing the Earth with a separation angle of $68^{\circ}$. OSCAR is designed to answer fundamental questions behind the trigger of CMEs in the lower solar corona, as well as to set up a space weather forecasting system for geo-effective CMEs and CIRs. The advantage of OSCAR resides in the originality of its design that enables to tackle those two goals simultaneously at moderate cost. We furthermore detailed in this work the basic analyses for the feasibility of the OSCAR mission. We put a particular emphasis on showing that, thanks to significant heritage, such a mission requires fairly small instrument developments (the main challenge resides in producing a sufficiently light EUV imager of the lower corona) to lead to important improvements in our scientific understanding of space weather events. In addition, we sketched a full spacecraft design and proposed very simple orbital phases to achieve the required constant angular separation of $68^{\circ}$ of the two spacecraft (see Fig. 2). In spite of the large distances involved, the telemetry needed for our mission is accessible with today's terrestrial infrastructures. It must be noted that even though the telemetry requirements may seem demanding, OSCAR would produce the necessary data for very valuable near-real time forecasts of the most dangerous space weather events. In conclusions, the design of the OSCAR mission includes for the very first time real-time predictive capabilities and provides a strong basis for the development of future space weather missions.
Acknowledgements. We thank the referees for constructive reports on our work. We acknowledge gratefully the Alpbach Summer School organisers and tutors for their invaluable help in the process of developing the concept of OSCAR. We acknowledge as well our financial support to participate in the Alpbach Summer School 2013: Centre National d'Etudes Spatiales - CNES (AS and TP), Deutsches Zentrum für Luft- und Raumfahrt - DLR (NJ, AM and MB), UK Space Agency - UKSA (AL), FFG - Aeronautics and Space Agency (PL), FFG-Austrian Research Promotion Agency (BS), Finnish Doctoral Programme in Astronomy and Space Physics (SH), Royal Observatory of Belgium (EK), Italian Space Agency ASI (SS), Danish Ministry of Higher Education and Science (SM), Enterprise Ireland (LO), Ministerio Español de Economía y Competitividad (VPB), Norwegian Space Center - NSC (CS). The editor thank two anonymous referees for their assistance in evaluating this paper.

\section{References}

Aschwanden, M.J., J.-P. Wülser, N. Nitta, and J. Lemen. Solar stereoscopy with STEREO/EUVI A and B spacecraft from small (6) to large (170) spacecraft separation angles. Sol. Phys., 281, 101-119, 2012, DOI: 10.1007/s11207-012-0092-8.

Bemporad, A. Stereoscopic reconstruction from STEREO/EUV imagers data of the three-dimensional shape and expansion of an erupting prominence. Astrophys. J., 701, 298-305, 2009, DOI: 10.1088/0004-637X/701/1/298.

Borovsky, J.E., and M.H. Denton. Differences between CME-driven storms and CIR-driven storms. J. Geophys. Res., 111, 7, 2006, DOI: 10.1029/2005JA011447.

Carr, C.M., T.S. Horbury, A. Balogh, W. Baumjohann, B. Bavassano, et al. A magnetometer for the solar orbiter mission. Proceedings of the Second Solar Orbiter Workshop, 41, ISBN: 92-9291-205-2, 2007.

Chen, P.F. Coronal mass ejections: models and their observational basis. Living Rev. Sol. Phys., 8, 1, 2011 DOI: $10.12942 / \mathrm{lrsp}-2011-1$.

Dal Lago, A., W.D. Gonzalez, A. De Lucas, C.R. Braga, L.R. Vieira, T.R.C. Stekel, and M. Rockenbach. CME dynamics using coronagraph and interplanetary ejecta data. Adv. Space Res., 51, 1942-1948, 2013, DOI: 10.1016/j.asr.2012.11.023.

Davies, J.A., C.H. Perry, R.M.G.M. Trines, R.A. Harrison, N. Lugaz, C. Möstl, Y.D. Liu, and K. Steed. Establishing a stereoscopic technique for determining the kinematic properties of solar wind transients based on a generalized self-similarly expanding circular geometry. Astrophys. J., 777, 167, 2013, DOI: $10.1088 / 0004-637 X / 777 / 2 / 167$.

Davis, C.J., C.A. de Koning, J.A. Davies, D. Biesecker, G. Millward, et al. A comparison of space weather analysis techniques used to predict the arrival of the Earth-directed CME and its shockwave launched on 8 April 2010. Space Weather, 9, S01005, 2011, DOI: $10.1029 / 2010$ SW000620.

Fan, Y. Magnetic fields in the solar convection zone. Living Rev. Sol. Phys., 6, 4, 2009, DOI: 10.12942/lrsp-2009-4.

Frazin, R.A., and F. Kamalabadi. On the use of total brightness measurements for tomography of the solar corona. Astrophys. J., 628, 1061-1069, 2005, DOI: 10.1086/430846.

Gandorfer, A., S.K. Solanki, J. Woch, V. Martnez Pillet, A. Álvarez Herrero, and T. Appourchaux. The Solar Orbiter Mission and its Polarimetric and Helioseismic Imager (SO/PHI). J. Phys.: Conf. Ser., 271, 2086, 2011, DOI: 10.1088/1742-6596/271/1/012086.

Gloeckler, G., J. Cain, F.M. Ipavich, E.O. Tums, P. Bedini, et al. Investigation of the composition of solar and interstellar matter using solar wind and pickup ion measurements with SWICS and SWIMS on the ACE spacecraft. Space. Sci. Rev., 86, 497-539, 1998, DOI: 10.1023/A:1005036131689.

Gold, R.E., S.M. Krimigis, S.E. HawkinsIII, D.K. Haggerty, D.A. Lohr, E. Fiore, T.P. Armstrong, G. Holland, and L.J. Lanzerotti. Electron, proton, and alpha monitor on the advanced composition explorer spacecraft. Space Sci. Rev., 86, 541-562, 1998, DOI: $10.1023 / \mathrm{A}: 1005088115759$. 
Gómez-Herrero, R., O. Malandraki, N. Dresing, E. Kilpua, B. Heber, A. Klassen, R. Müller-Mellin, and R.F. WimmerSchweingruber. Spatial and temporal variations of CIRs: Multipoint observations by STEREO. J. Atmos. Sol. Terr. Phys., 73, 551-565, 2011, DOI: 10.1016/j.jastp.2010.11.017.

Gopalswamy, N., A. Lara, R.P. Lepping, M.L. Kaiser, D. Berdichevsky, and O.C. St Cyr. Interplanetary acceleration of coronal mass ejections. Geophys. Res. Lett., 27, 145-148, 2000, DOI: 10.1029/1999GL003639.

Gosling, J.T. In-Situ observations of coronal mass ejections in interplanetary space. In: Eruptive Solar Flares. Proceedings of Colloquium \#133 of the International Astronomical Union, Berlin, Heidelberg, Springer Berlin Heidelberg, 258-267, 1992, ISBN 978-3-540-55246-8. DOI: 10.1007/3-540-55246-4_107.

Howard, R.A., J.D. Moses, A. Vourlidas, J.S. Newmark, D.G. Socker, et al. Sun Earth Connection Coronal and Heliospheric Investigation (SECCHI), Space. Sci. Rev., 136, 67-115, 2008, DOI: $10.1007 / \mathrm{s} 11214-008-9341-4$.

Howard, T.A., and S.J. Tappin. Three-dimensional reconstruction of two solar coronal mass ejections using the STEREO spacecraft. Sol. Phys., 252, 373-383, 2008, DOI: 10.1007/s11207-008-9262-0.

Kahler, S.W., H. Aurass, G. Mann, and A. Klassen. The production of near-relativistic electrons by CME-driven shocks. Coronal and Stellar Mass Ejections, 226, 338-345, 2005, DOI: $10.1017 / \mathrm{S} 1743921305000839$

Liu, Y.D., J.G. Luhmann, P. Kajdič, E.K.J. Kilpua, N. Lugaz, et al. Observations of an extreme storm in interplanetary space caused by successive coronal mass ejections. Nature Communications, $\mathbf{5}$, 3481, 2014, DOI: 10.1038/ncomms4481.

Lugaz, N., P. Kintner, C. Möstl, L.K. Jian, C.J. Davis, and C.J. Farrugia. Heliospheric observations of STEREO-directed coronal mass ejections in 2008-2010: Lessons for future observations of earth-directed CMEs. Sol. Phys., 279, 497-515, 2012, DOI: $10.1007 / \mathrm{s} 11207-012-0007-8$.

Manoharan, P.K. Evolution of coronal mass ejections in the inner heliosphere: A study using white-light and scintillation images. Sol. Phys., 235, 345-368, 2006,

DOI: $10.1007 / \mathrm{s} 11207-006-0100-y$.

Manoharan, P.K. and A. Mujiber Rahman, Coronal mass ejections Propagation time and associated internal energy, J. Atmos. Sol. Terr. Phys., 73, 671-677, 2011, DOI: $10.1016 /$ j.jastp.2011.01.017.

Mason, G.M., M.I. Desai, U. Mall, A. Korth, R. Bucik, T.T. von Rosenvinge, and K.D. Simunac. In situ observations of CIRs on STEREO, wind, and ACE during 2007-2008. Sol. Phys., 256, 393-408, 2009, DOI: 10.1007/s11207-009-9367-0.

McComas, D.J., S.J. Bame, P. Barker, W.C. Feldman, J.L. Phillips, P. Riley, and J.W. Griffee. Solar Wind Electron Proton Alpha Monitor (SWEPAM) for the advanced composition explorer. Space Sci. Rev., 86, 563-612, 1998, DOI: $10.1023 / \mathrm{A}: 1005040232597$.

Möstl, C., K. Amla, J.R. Hall, P.C. Liewer, E.M. De Jong, et al. Connecting speeds, directions and arrival times of 22 coronal mass ejections from the Sun to 1 Au. Astrophys. J., 787, 119, 2014, DOI: $10.1088 / 0004-637 X / 787 / 2 / 119$.

Nitta, N.V., M.J. Aschwanden, S.L. Freeland, J.R. Lemen, J.P. Wülser, and D.M. Zarro. The association of solar flares with coronal mass ejections during the extended solar minimum. Sol. Phys., 289, 1257-1277, 2013, DOI: 10.1007/s11207-013-0388-3.

Pulkkinen, T. Space weather: terrestrial perspective. Living Rev. Sol. Phys., 4, 1, 2007, DOI: 10.12942/lrsp-2007-1.
Richardson, I.G. Energetic particles and corotating interaction regions in the solar wind. Space Sci. Rev., 111, 267-376, 2004, DOI: 10.1023/B:SPAC.0000032689.52830.3e.

Rouillard, A.P., N.R. Sheeley, A. Tylka, A. Vourlidas, C.K. Ng, et al. The longitudinal properties of a solar energetic particle event investigated using modern solar imaging. Astrophys. J., 752, 44, 2012, DOI: 10.1088/0004-637X/752/1/44.

Sauvaud, J.A., D. Larson, C. Aoustin, D. Curtis, J.L. Médale, et al. The IMPACT Solar Wind Electron Analyzer (SWEA). Space Sci. Rev., 136, 227-239, 2008, DOI: 10.1007/s11214-007-9174-6.

Schwenn, R. Space weather: the solar perspective. Living Rev. Sol. Phys., 3, 2, 2006, DOI: 10.12942/lrsp-2006-2.

Shibata, K., and T. Magara. Solar flares: magnetohydrodynamic processes. Living Rev. Sol. Phys., 8, 6, 2011. DOI: 10.12942/lrsp-2011-6.

Simnett, G.M., E.C. Roelof, and D.K. Haggerty. The acceleration and release of near-relativistic electrons by coronal mass ejections. Astrophys. J., 579, 854-862, 2002, DOI: $10.1086 / 342871$.

Smith, Z., W. Murtagh, and C. Smithtro. Relationship between solar wind low-energy energetic ion enhancements and large geomagnetic storms. J. Geophys. Res., 109, 1110, 2004, DOI: 10.1029/2003JA010044.

Stone, E.C., A.M. Frandsen, R.A. Mewaldt, E.R. Christian, D. Margolies, J.F. Ormes, and F. Snow. The advanced composition explorer. Space Sci. Rev., 86, 1-22, 1998, DOI: $10.1023 / \mathrm{A}: 1005082526237$.

Tang, Y.Q., and G.M. Le. Statistical analysis of soft X-ray flares during the 23rd Solar Cycle. International Cosmic Ray Conference, 1, 5, 2005.

Tappin, S.J., and T.A. Howard. Direct observation of a corotating interaction region by three spacecraft. Astrophys. J., 702, 862-870, 2009, DOI: 10.1088/0004-637X/702/2/862.

Webb, D.F., and T.A. Howard. Coronal mass ejections: observations. Living Rev. Sol. Phys., 9, 3, 2012, DOI: 10.12942/lrsp-2012-3.

Wertz, J.R., and W.J. Larson. Space mission analysis and design. Microcosm, 3rd illustrated edn. Kluwer, 2003.

Yashiro, S., N. Gopalswamy, S. Akiyama, G. Michalek, and R.A. Howard. Visibility of coronal mass ejections as a function of flare location and intensity. J. Geophys. Res., 110, 12, 2005, DOI: $10.1029 / 2005$ JA011151.

Yashiro, S., N. Gopalswamy, G. Michalek, O.C. St Cyr, S.P. Plunkett, N.B. Rich, and R.A. Howard. A catalog of white light coronal mass ejections observed by the SOHO spacecraft. J. Geophys. Res., 109, 7105, 2004, DOI: 10.1029/2003JA010282.

Zhang, J., I.G. Richardson, D.F. Webb, N. Gopalswamy, E. Huttunen, et al. Solar and interplanetary sources of major geomagnetic storms (Dst $\leq-100 \mathrm{nT}$ ) during 1996-2005. J. Geophys. Res., 112, 10,102, 2007, DOI: 10.1029/2007JA012321.

Zhukov, A.N., and F. Auchere. On the nature of EIT waves, EUV dimmings and their link to CMEs. $A \& A, 427,705-716,2004$, DOI: 10.1051/0004-6361:20040351.

Zuccarello, F., L. Balmaceda, G. Cessateur, H. Cremades, S.L. Guglielmino, et al. Solar activity and its evolution across the corona: recent advances. J. Space Weather Space Clim., 3, 18, 2013, DOI: $10.1051 / \mathrm{swsc} / 2013039$.

Zwickl, R.D., K.A. Doggett, S. Sahm, W.P. Barrett, R.N. Grubb, et al. The NOAA Real-Time Solar-Wind (RTSW) system using ACE data. Space Sci. Rev., 86, 633-648, 1998, DOI: $10.1023 / \mathrm{A}: 1005044300738$.

Cite this article as: Strugarek A., N. Janitzek, A. Lee, P. Löschl, B. Seifert, et al. A Space Weather mission concept: Observatories of the Solar Corona and Active Regions (OSCAR). J. Space Weather Space Clim., 5, A4, 2015, DOI: 10.1051/swsc/2015003. 